\title{
Genomic Organization and Expression of Iron Metabolism Genes in the Emerging Pathogenic Mold Scedosporium apiospermum
}

\author{
Yohann Le Govic ${ }^{1,2 *}$, Nicolas Papon ${ }^{1}$, Solène Le Gal ${ }^{3,4}$, Bénédicte Lelièvre ${ }^{1,5}$, \\ Jean-Philippe Bouchara ${ }^{1,2}$ and Patrick Vandeputte ${ }^{1,2}$ \\ 1 Groupe d'Etude des Interactions Hôte-Pathogène (EA 3142), SFR ICAT 4208, UNIV Angers, UNIV Brest, Angers, France, \\ ${ }^{2}$ Laboratoire de Parasitologie-Mycologie, Centre Hospitalier Universitaire, Angers, France, ${ }^{3}$ Groupe d'Etude des Interactions \\ Hôte-Pathogène (EA 3142), SFR ICAT 4208, UNIV Angers, UNIV Brest, Brest, France, ${ }^{4}$ Laboratoire de \\ Parasitologie-Mycologie, Centre Hospitalier Universitaire, Brest, France, ${ }^{5}$ Laboratoire de Pharmacologie-Toxicologie, Centre \\ Hospitalier Universitaire, Angers, France
}

\section{OPEN ACCESS}

Edited by:

Joshua D. Nosanchuk, Albert Einstein College of Medicine, United States

Reviewed by: Tamás Papp,

University of Szeged, Hungary Marcio L. Rodrigues, Fundação Oswaldo Cruz (Fiocruz),

Brazil

*Correspondence: Yohann Le Govic yohann.legovic@chu-angers.fr

Specialty section: This article was submitted to Fungi and Their Interactions, a section of the journal Frontiers in Microbiology

Received: 22 January 2018 Accepted: 11 April 2018 Published: 26 April 2018

Citation:

Le Govic Y, Papon N, Le Gal S, Lelièvre B, Bouchara J-P and Vandeputte P (2018) Genomic Organization and Expression of Iron Metabolism Genes in the Emerging Pathogenic Mold Scedosporium apiospermum. Front. Microbiol. 9:827. doi: 10.3389/fmicb.2018.00827
The ubiquitous mold Scedosporium apiospermum is increasingly recognized as an emerging pathogen, especially among patients with underlying disorders such as immunodeficiency or cystic fibrosis (CF). Indeed, it ranks the second among the filamentous fungi colonizing the respiratory tract of CF patients. However, our knowledge about virulence factors of this fungus is still limited. The role of iron-uptake systems may be critical for establishment of Scedosporium infections, notably in the iron-rich environment of the CF lung. Two main strategies are employed by fungi to efficiently acquire iron from their host or from their ecological niche: siderophore production and reductive iron assimilation (RIA) systems. The aim of this study was to assess the existence of orthologous genes involved in iron metabolism in the recently sequenced genome of $S$. apiospermum. At first, a tBLASTn analysis using $A$. fumigatus iron-related proteins as query revealed orthologs of almost all relevant loci in the $S$. apiospermum genome. Whereas the genes putatively involved in RIA were randomly distributed, siderophore biosynthesis and transport genes were organized in two clusters, each containing a non-ribosomal peptide synthetase (NRPS) whose orthologs in A. fumigatus have been described to catalyze hydroxamate siderophore synthesis. Nevertheless, comparative genomic analysis of siderophore-related clusters showed greater similarity between S. apiospermum and phylogenetically close molds than with Aspergillus species. The expression level of these genes was then evaluated by exposing conidia to iron starvation and iron excess. The expression of several orthologs of $A$. fumigatus genes involved in siderophore-based iron uptake or RIA was significantly induced during iron starvation, and conversely repressed in iron excess conditions. Altogether, these results indicate that $S$. apiospermum possesses the genetic information required for efficient and competitive iron uptake. They also suggest an important role of the siderophore production system in iron uptake by $S$. apiospermum.

Keywords: Scedosporium, genome mining, iron, siderophore, gene expression 


\section{INTRODUCTION}

Iron is the fourth most common element found on the Earth's crust (Frey and Reed, 2012). However, in spite of its abundance, iron is fairly accessible to living organisms as a result of its very limited solubility under aerobic conditions. Iron is mainly encountered in two relatively stable oxidation states, ferrous (Fe(II) or $\mathrm{Fe}^{2+}$ ) and ferric ( $\mathrm{Fe}(\mathrm{III})$ or $\mathrm{Fe}^{3+}$ ). Due to the reversible switching between the $\mathrm{Fe}^{2+}$ and $\mathrm{Fe}^{3+}$ species and its ability to form coordination complexes with organic ligands, iron plays a critical role in numerous biochemical processes including oxidative phosphorylation, DNA replication, and biosynthesis of small molecules such as lipids, amino acids, and sterols (Philpott, 2006). On other hand, iron excess can be harmful to the cell owing to its capacity to catalyze the formation of reactive oxygen species (ROS) and to initiate lipid peroxidation (Halliwell and Gutteridge, 1984). Therefore, microbes have evolved sophisticated systems to overcome suboptimal iron availability and meanwhile to prevent iron overload toxicity.

Four distinct mechanisms of iron acquisition have been described in fungi: (i) heme uptake and degradation, (ii) lowaffinity ferrous iron uptake, which occurs through relatively non-specific divalent cation transporters, (iii) reductive iron assimilation (RIA), employing a high-affinity uptake system in which ferrous iron is first oxidized by a multicopperferroxidase before being transferred to the cytosol via a specific $\mathrm{Fe}(\mathrm{III})$-permease, and (iv) siderophore-mediated iron uptake (Haas, 2014). Siderophores are amongst the strongest natural $\mathrm{Fe}$ (III)-chelating products. The majority of fungal siderophores belongs to the hydroxamate class and can be divided into four structural families: rhodotorulic acid, fusarinines, coprogens, and ferrichromes. The hydroxamate functional group is synthesized from L-ornithine, a non-proteinogenic amino acid that is produced either in mitochondria from L-glutamate or in the cytosol through hydrolysis of L-arginine (Schafferer et al., 2015). The first key enzyme of the hydroxamate biosynthetic pathway is the L-ornithine- $N^{5}$-monooxygenase SidA, which catalyzes $N^{5}$-hydroxylation of L-ornithine (Eisendle et al., 2003). The hydroxamate motif is then formed by $N^{5}$ acylation of $N^{5}$-hydroxy-L-ornithine by $N^{5}$-transacylases. Here, the pathway splits as different acyl groups can be attached to hydroxyornithine, defining the nature of the siderophore produced. In Aspergillus fumigatus, this step is mediated by two transacetylases: SidL, which adds an acetyl group in ferrichrometype siderophores (Blatzer et al., 2011c), and SidF, which adds an anhydromevalonyl group in siderophores of the coprogen and fusarinine families (Schrettl et al., 2007). AnhydromevalonylCoA is obtained from mevalonate by consecutive CoA-ligation and dehydration catalyzed by the peroxisomal enzymes SidI and SidH, respectively (Yasmin et al., 2012; Gründlinger et al., 2013). The ultimate step consists in the covalent linkage of the $N^{5}$ acyl- $N^{5}$-hydroxy-L-ornithine groups, and is orchestrated by nonribosomal peptide synthetases (NRPSs). After being activated by a $4^{\prime}$-phosphopantetheinyltransferase protein (NpgA/PptA), the NRPSs SidC and SidD achieve assembly of intra- and extracellular siderophores in A. fumigatus, respectively (Schrettl et al., 2007). Excreted siderophores bind Fe(III) ions to form ferrisiderophores chelates, which are then imported into the cell through specific plasma membrane-localized transporters termed "siderophore-iron transporters" (SITs). At least two types of SITs have been identified in the aspergilli, including Aspergillus SitT and MirA-D proteins, which belong to the ATP-binding cassette and major facilitator superfamilies, respectively (Haas et al., 2003; Schrettl et al., 2008, 2010). Once entered into the cytoplasm, iron is released from siderophores and finally becomes available for various cellular processes.

In addition to the non-reductive siderophore-mediated iron acquisition, pathogenic fungi developed a reductive, nonchelating high affinity iron uptake system called "reductive iron assimilation" (RIA). RIA necessitates the reduction by a ferric reductase (Fre family) of highly insoluble ferric iron to more soluble and bioavailable ferrous iron, combined with a specific transport system composed of a multicopper ferroxidase (Fet family) associated with a ferric permease (Ftr family) (Kosman, 2013). The Fet and Ftr proteins are inextricably linked together since they are assembled into a stable complex prior to plasma membrane trafficking (Stearman et al., 1996). Indeed, the Fetmediated Fe(II) oxidation step is mandatory to the permeation step, i.e., the Ftr channel only accepts Fe(III) generated by the coupling Fet protein (Wang et al., 2003). Unlike Cryptococcus neoformans and Candida albicans ferric permeases, A. fumigatus FtrA has been demonstrated to be dispensable for fungal virulence, in the presence of a functional siderophore iron uptake system (Ramanan and Wang, 2000; Schrettl et al., 2004; Jung et al., 2008). Furthermore, ferric reductases play an important role in the removal of iron from siderophores (Yun et al., 2001) or from host iron sources, such as heme and transferrin (Knight et al., 2005; Saikia et al., 2014).

In A. fumigatus, optimal iron balance is maintained by two central regulatory proteins, which are interconnected in a negative feedback loop: the GATA-transcription factor SreA and the bZIP-transcription factor HapX (Haas, 2014). During iron starvation, HapX represses iron-consuming pathways (e.g., heme biosynthesis and respiration) and activates siderophore production through interaction with the CCAAT-binding complex (CBC). On the other hand, during iron sufficiency, SreA down-regulates both RIA and siderophore uptake systems via binding to the consensus DNA sequence ATCWGATAA. The disruption of hapX, but not of sreA, was shown to impair virulence of $A$. fumigatus in murine models of invasive aspergillosis (Schrettl et al., 2008, 2010). These observations highlight the need to adapt to iron limitation for establishing fungal infection, which is consistent with the fact that intraand extracellular siderophores play a pivotal role in A. fumigatus virulence (Schrettl et al., 2007).

Scedosporium apiospermum is a ubiquitous fungus capable of causing a wide range of infections in human (Cortez et al., 2008). Despite numerous studies showing an increasing health threat, especially among patients with underlying conditions (e.g., immunodeficiency or cystic fibrosis) (Walsh and Groll, 1999; Guarro et al., 2006; Lamaris et al., 2006; Pihet et al., 2009; Douglas et al., 2016; Koehler et al., 2016; Chen et al., 2017), little is known about virulence factors enabling the fungus to produce acute or chronic infections. Moreover, Scedosporium 
infections are extremely difficult to treat due to the high level of intrinsic resistance to many, if not all, of current antifungals (Cortez et al., 2008). To gain insight into the pathogenic and drug resistance mechanisms of this fungus, the genome of a clinical isolate of S. apiospermum was fully sequenced in 2014 (Vandeputte et al., 2014). Here, we describe the first genomic and transcriptional analysis focusing on genes related to iron metabolism in S. apiospermum.

\section{MATERIALS AND METHODS}

\section{Strain and Culture Conditions}

Scedosporium apiospermum (S. apiospermum; taxid:563466) whole-genome sequenced strain IHEM 14462, originally isolated from a sputum sample from a cystic fibrosis patient (Vandeputte et al., 2014), was grown on Potato Dextrose Agar (Conda, Madrid, Spain) plates at $37^{\circ} \mathrm{C}$ for 9 days to induce sporulation. Conidia were harvested from colonies by aseptically scraping the plates using $1 \mathrm{X}$ TE buffer $(10 \mathrm{mM}$ Tris(hydroxymethyl)aminomethane, $\mathrm{HCl} \mathrm{pH} \mathrm{7.5,} 1 \mathrm{mM}$ EDTA $\mathrm{NaOH} \mathrm{pH}$ 8) and passing through Miracloth ${ }^{\circledR}$ mesh filter (Merck, Darmstad, Germany) to remove the mycelia. The filtrate was centrifuged $(4,600 \mathrm{rpm}, 5 \mathrm{~min})$ and pelleted conidia were resuspended in $1 \mathrm{X}$ TE buffer. Conidia were numbered with a hemocytometer and a total of $10^{7}$ conidia were inoculated into $100-\mathrm{ml}$ flasks containing $25 \mathrm{ml}$ of YEPD medium (containing per liter: $5 \mathrm{~g}$ yeast extract, $10 \mathrm{~g}$ peptone, $20 \mathrm{~g}$ dextrose, and $0.5 \mathrm{~g}$ chloramphenicol). Cultures were incubated for $48 \mathrm{~h}$ at $37^{\circ} \mathrm{C}$ with agitation $(120 \mathrm{rpm})$. Iron excess was obtained by supplementing YEPD medium with $20 \mu \mathrm{M}$ of either free $\left(\mathrm{FeSO}_{4}\right.$ or $\mathrm{FeCl}_{3}$ ) or transferrin-bound iron (holotransferrin, Thermo Fisher, Karlsruhe, Germany). Iron-depleted conditions were obtained by adding $200 \mu \mathrm{M}$ bathophenanthroline disulfonate (BPS, Sigma-Aldrich, Saint-Quentin Fallavier, France) in YEPD medium.

\section{Genome Mining}

Identification of $S$. apiospermum genes potentially involved in iron metabolism was performed as described by Haas (Haas, 2012), searching for orthologs of A. fumigatus strain Af293 (A. fumigatus; taxid:330879) iron-related proteins through tBLASTn analysis (https://blast.ncbi.nlm.nih.gov/Blast.cgi) against $S$. apiospermum genome. Only results corresponding to strain IHEM 14462 with an $e$-value $<1$ e-15 on at least $40 \%$ of the query sequence were considered. Organization into clusters of the genes found in S. apiospermum genome was further compared with those identified in the genomes of $A$. fumigatus strain Af293 (Nierman et al., 2005), A. nidulans strain FGSC A4 (taxid:227321) (Galagan et al., 2005), A. niger strain CBS 513.88 (taxid:425011) (Pel et al., 2007), Colletotrichum higginsianum strain IMI 349063 (taxid:759273) (O’Connell et al., 2012) and Trichoderma reesei strain QM6a (taxid:431241) (Martinez et al., 2008). Searching for putative binding sites of the transcription factor HapX (Hortschansky et al., 2007) was performed within the $2 \mathrm{~kb}$ upstream region of each gene putatively involved in RIA and siderophore metabolism by using the MEME Suite's FIMO (Grant et al., 2011).

\section{RNA Isolation, Retrotranscription and Real-Time Quantitative PCR}

Fungal cells from triplicate cultures in standard, iron-overloaded and iron-depleted conditions were harvested at $48 \mathrm{~h}$ and ground in liquid nitrogen with a mortar and pestle. Total RNA was recovered by processing the fungal powder with the NucleoSpin ${ }^{\circledR}$ RNA Plant kit (Macherey-Nagel, Düren, Germany), according to the manufacturer's instructions. All RNA samples $(5 \mu \mathrm{g})$ were treated with $2 \mathrm{U}$ of RNase-free DNase I (Ambion ${ }^{\mathrm{TM}}$ Life Technologies, Carlsbad, CA), according to the protocol supplied by the manufacturer. Complementary DNA were synthesized from $500 \mathrm{ng}$ total RNA using SuperScript IV reverse transcriptase (200 U; Invitrogen Life Technologies, Carlsbad, CA) in the presence of oligo-d(T) primer $(2.5 \mu \mathrm{M})$, deoxyribonucleoside triphosphates $(0.5 \mathrm{mM}$ each), dithiotreitol ( $5 \mathrm{mM})$, and RNase inhibitor (2 U). Thereafter, cDNA were 20fold diluted and used as template for real-time quantitative PCR (qPCR). Each reaction (12.5 $\mu \mathrm{l}$ final volume) contained Fast SYBR $^{\circledR}$ Green PCR Master Mix (Applied Biosystems, Foster City, CA), $200 \mathrm{nM}$ of each primer (Integrated DNA Technologies Inc., Leuven, Belgium), and $1 \mu \mathrm{l}$ of diluted cDNA. Primers used to perform qPCR experiments are compiled in Supplementary Table S1. qPCR reactions were carried out on a StepOnePlus ${ }^{\mathrm{TM}}$ thermocycler (Applied Biosystems) with the following thermal profile: $95^{\circ} \mathrm{C}$ for $2 \mathrm{~min}, 40$ cycles of $95^{\circ} \mathrm{C}$ for $3 \mathrm{~s}, 60^{\circ} \mathrm{C}$ for $30 \mathrm{~s}$. Melting curve analysis was performed immediately after the amplification procedure as follows: $95^{\circ} \mathrm{C}$ for $15 \mathrm{~s}$, and stepwise annealing from 60 to $94.9^{\circ} \mathrm{C}$ with $0.3^{\circ} \mathrm{C}$ increments. For each gene, fold changes relative to standard condition (i.e., YEPD medium) were calculated in each condition using the deltadelta $\mathrm{Ct}$ method and $u b c B$ and sarA genes as endogenous controls (Llanos et al., 2015). For each data point, three biological replicates and two technical replicates were performed, and the variation in expression of a given gene was considered significant if the $\log 2$ fold change \pm standard deviation was $>1$ or $<-1$.

\section{RESULTS}

\section{Genome Mining for Iron Homeostasis in Scedosporium apiospermum}

Computational identification of genes putatively involved in iron metabolism in $S$. apiospermum was performed through a tBLASTn analysis, using A. fumigatus Af293 iron-related proteins as query (Haas, 2012). This strategy allowed to find orthologs of all genes involved in iron acquisition and storage (Table 1), with the exception of $s r b A$, which encodes a regulatory protein that activates iron uptake during iron deprivation (Blatzer et al., 2011a), sidG, which encodes a protein that catalyzes fusarinine $C$ esterification, and $e s t B$ and sidJ, which both encode proteins involved in triacetylfusarinine $\mathrm{C}$ saponification (Kragl et al., 2007; Schrettl et al., 2007). Furthermore, this analysis revealed that $S$. apiospermum genome contains two putative gene clusters harboring an iron-related NRPS as the core member (Figure 1). Indeed, the closest orthologs of these NRPS genes, sidC and sidD, are known or presumed to be involved in siderophore synthesis in A. fumigatus (Haas, 2012), A. nidulans (von Döhren, 2009), 
TABLE 1 | Results of tBLASTn analysis of the genes putatively involved in iron metabolism in S. apiospermum against A. fumigatus Af293 (taxid: 330879 ).

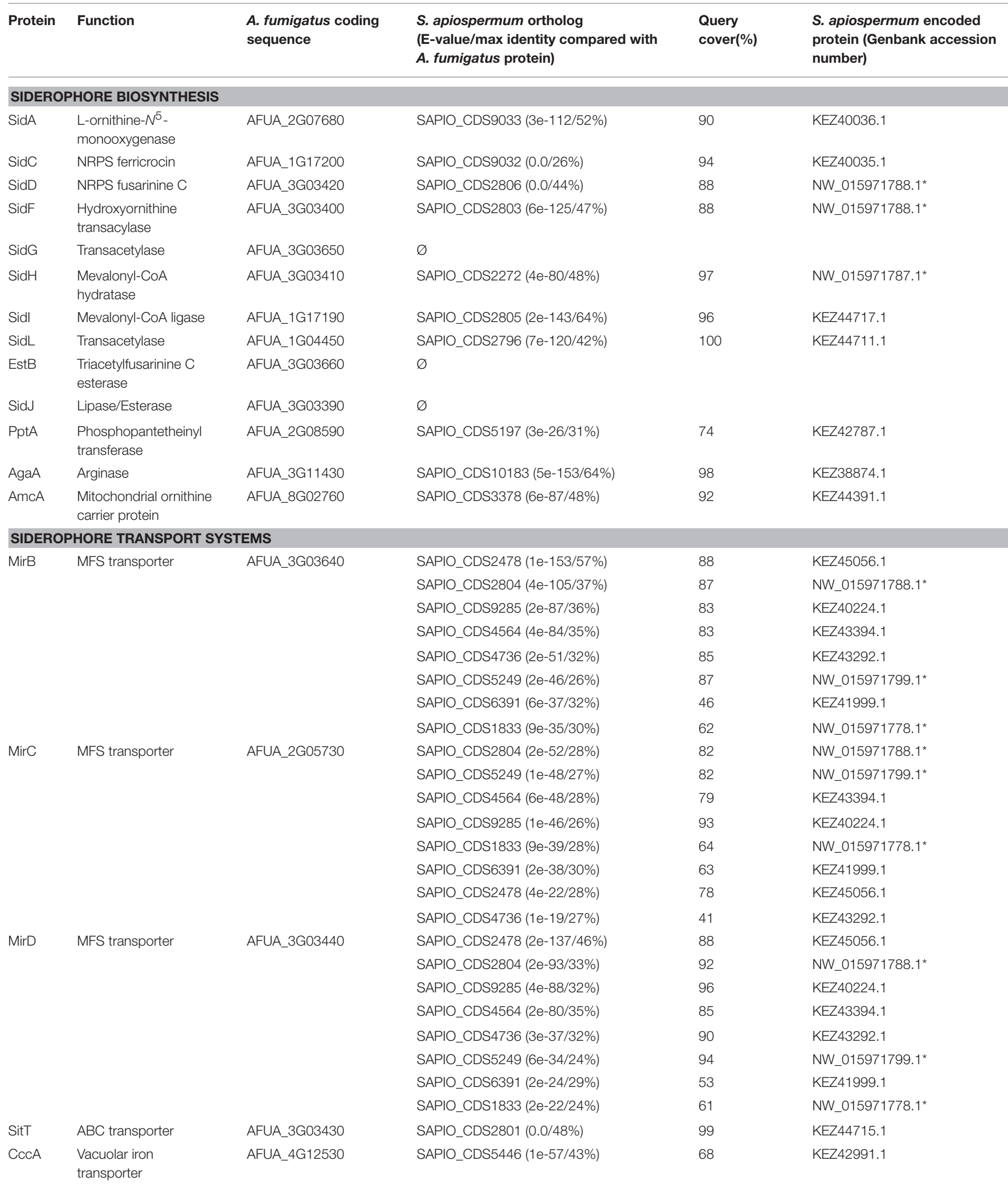


TABLE 1 | Continued

\begin{tabular}{|c|c|c|c|c|c|}
\hline Protein & Function & $\begin{array}{l}\text { A. fumigatus coding } \\
\text { sequence }\end{array}$ & $\begin{array}{l}\text { S. apiospermum ortholog } \\
\text { (E-value/max identity compared with } \\
\text { A. fumigatus protein) }\end{array}$ & $\begin{array}{l}\text { Query } \\
\text { cover(\%) }\end{array}$ & $\begin{array}{l}\text { S. apiospermum encoded } \\
\text { protein (Genbank accession } \\
\text { number) }\end{array}$ \\
\hline \multicolumn{6}{|c|}{ REGULATORY PROTEINS } \\
\hline AcuM & $\begin{array}{l}\text { Zn2Cys6 transcription } \\
\text { factor }\end{array}$ & AFUA_2G12330 & SAPIO_CDS0915 (7e-79/48\%) & 65 & KEZ46068.1 \\
\hline MpkA & MAP kinase $A$ & AFUA_4G13720 & SAPIO_CDS2689 (1e-162/73\%) & 99 & KEZ45223.1 \\
\hline PacC & $\begin{array}{l}\text { Cys2His2 transcription } \\
\text { factor }\end{array}$ & AFUA_3G11970 & SAPIO_CDS0213 (5e-42/66\%) & 69 & KEZ46879.1 \\
\hline SreA & $\begin{array}{l}\text { ZnF_GATA transcription } \\
\text { factor }\end{array}$ & AFUA_5G11260 & SAPIO_CDS7310 (1e-34/39\%) & 40 & KEZ41223.1 \\
\hline SrbA & $\begin{array}{l}\text { bHLH transcription } \\
\text { factor }\end{array}$ & AFUA_2G01260 & $\varnothing$ & & \\
\hline HapX & $\begin{array}{l}\text { bZip transcription } \\
\text { factor }\end{array}$ & AFUA_5G03920 & SAPIO_CDS9738 (8e-22/30\%) & 49 & NW_015971844.1* \\
\hline \multicolumn{6}{|c|}{ REDUCTIVE IRON ASSIMILATION } \\
\hline \multirow[t]{9}{*}{ FreB } & Ferric reductase & AFUA_1G17270 & SAPIO_CDS2383 (5e-67/38\%) & 75 & KEZ44995.1 \\
\hline & & & SAPIO_CDS1476 (1e-46/28\%) & 69 & KEZ45701.1 \\
\hline & & & SAPIO_CDS9014 (2e-39/30\%) & 48 & KEZ40025.1 \\
\hline & & & SAPIO_CDS10508 (4e-37/23\%) & 69 & KEZ39117.1 \\
\hline & & & SAPIO_CDS10060 (4e-30/24\%) & 70 & NW_015971855.1* \\
\hline & & & SAPIO_CDS9433 (1e-28/26\%) & 56 & KEZ39544.1 \\
\hline & & & SAPIO_CDS5404 (7e-24/24\%) & 69 & KEZ42955.1 \\
\hline & & & SAPIO_CDS6952 (2e-17/26\%) & 49 & NW_015971810.1* \\
\hline & & & SAPIO_CDS10726 (2e-17/23\%) & 41 & KEZ38703.1 \\
\hline \multirow[t]{3}{*}{ FetC } & Multicopper ferroxidase & AFUA_5G03790 & SAPIO_CDS0314 (2e-103/55\%) & 96 & KEZ46527.1 \\
\hline & & & SAPIO_CDS8659 (1e-62/51\%) & 87 & KEZ40718.1 \\
\hline & & & SAPIO_CDS0322 ${ }^{\#}(0.0 / 54 \%)^{\$}$ & & KEZ46534.1 \\
\hline \multirow[t]{2}{*}{ FtrA } & Iron permease & AFUA_5G03800 & SAPIO_CDS0321 (3e-95/52\%) & 94 & KEZ46533.1 \\
\hline & & & SAPIO_CDS0315\# $(2 \mathrm{e}-107 / 49 \%)^{\$}$ & & KEZ46528.1 \\
\hline
\end{tabular}

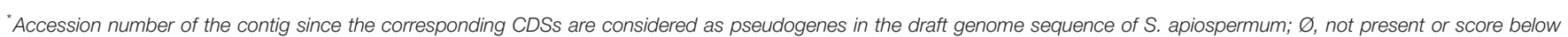

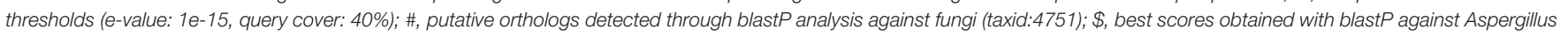
fumigatus Af293.

and A. niger (Franken et al., 2014). In S. apiospermum, the sidCrelated cluster contains an ortholog of sidA, which controls the initiation step of fungal hydroxamate siderophore biosynthesis (Eisendle et al., 2003). The second cluster is a combination made up of six genes putatively involved in siderophore production (i.e., sidD, sidF, sidI, and sidL orthologs) and transport (i.e., one sit $T$ and one mir orthologs). The sidH ortholog is located in a different region of the genome together with a putative MFS transporter gene (CDS2271), and is separated from the sidD cluster by $110 \mathrm{~kb}$. Data mining also revealed the existence of 7 other mir orthologs randomly distributed within S. apiospermum genome.

Of note, we found that the $S$. apiospermum sidL gene was not correctly annotated (Figure 2). Indeed, this coding region (CDS2796) is made of three exons - E1 (1,469 bp), E2 (600 bp), and E3 (277 bp) - and two introns - I1 (783 bp) and I2 (73 bp) - while in A. fumigatus and A. nidulans, sidL gene contains only 2 exons ( $\sim 1,400$ and $120 \mathrm{bp}$, respectively) separated by a $\sim 50$ bp intron. In other words, the $S$. apiospermum sidL ORF is twice longer than its Aspergillus orthologs, partly due to a long first intron. Interestingly, a Pfam analysis (http://pfam.xfam. org/) of the deduced protein sequence identified two conserved motifs in CDS2796: an acetyltransferase (GNAT) domain as expected for sidL, but also a cytochrome heme lyase domain. This discrepancy led us to refine the analysis of CDS2796 sequence which revealed the presence of previously undetected exonintron boundaries, one located at the $3^{\prime}$ end of E1, and another 96 bp away (i.e., inside I1), from which the transcription of a 120 bp supplementary exon occurs. The size of the mRNA transcribed from CDS2796 was further confirmed experimentally by designing 3 pairs of primers (Figure 2A). The first pair spans the newly discovered intron, and a PCR performed on cDNA with these primers amplified an expected $123 \mathrm{bp}$-fragment, which confirms the existence of this predicted intron (Figure 2B). The second pair of primers was designed to cover the last intron, and also amplified a fragment of the expected size. On the opposite, the third pair of primers, covering CDS2796 from start to stop codons as automatically annotated, gave no amplification, further confirming that the transcription of CDS2796 produces two mRNAs, the most upstream corresponding to a sidL ortholog, in 

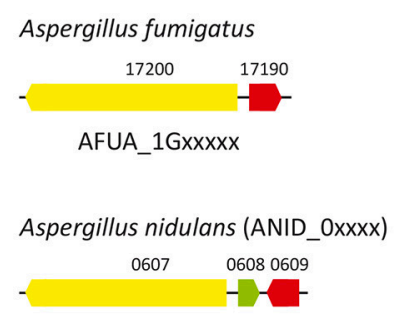
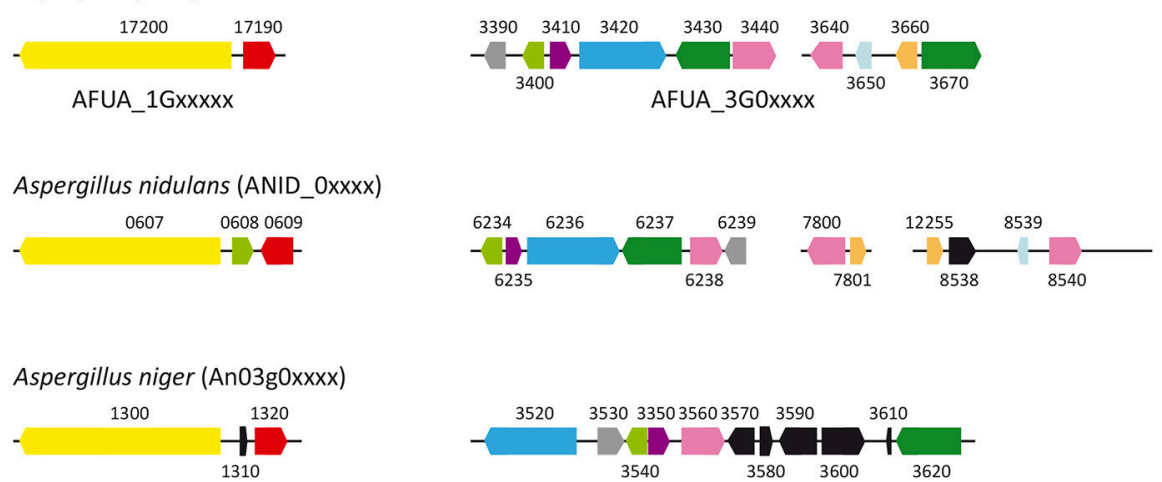

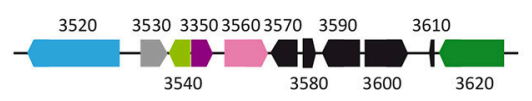

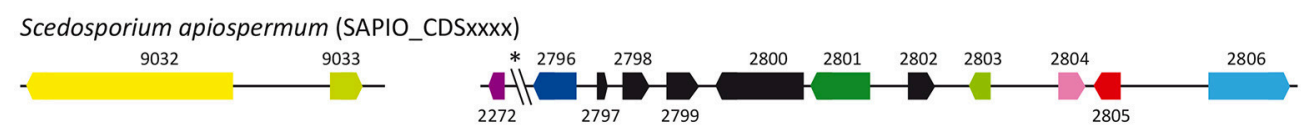
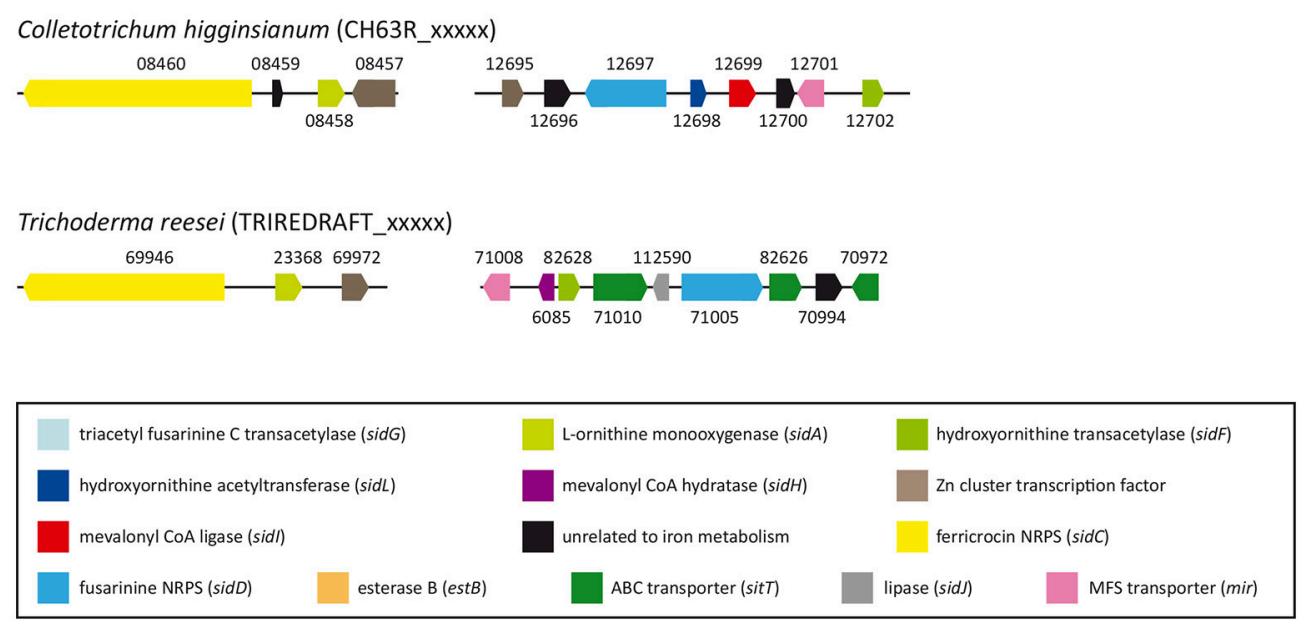

* the region between SAPIO_CDS2272 and SAPIO_CDS2796 spans about $110 \mathrm{~kb}$ and contains only genes unrelated to iron metabolism

FIGURE 1 | Distribution of siderophore biosynthetic and transporter genes in S. apiospermum IHEM 14462, A. fumigatus Af293, A. nidulans FGSC A4, A. niger CBS 513.88, C. higginsianum IMI 349063, and T. reesei QM6a genomes. Most of the siderophore-related genes are clustered within all the six filamentous fungi, but the content of these clusters varies from one species to another. Of note, gene organization in S. apiospermum is closer to that observed in $C$. higginsianum and T. reesei. Orthologous genes, taking A. fumigatus as reference, are identically colored. (adapted from Franken et al., 2014).

agreement with the sequence of this protein in Aspergillus species. The annotation of the contig containing the S. apiospermum sidL gene (GenBank accession number NW_015971788.1) has been updated accordingly.

Aside from siderophore-mediated iron acquisition gene battery, the tBLASTn analysis allowed the detection of several genes putatively involved in RIA. Indeed, two A. fumigatus fet $C$ and one $f t r A$ orthologs were identified in S. apiospermum. The fetC ortholog displaying the lowest similarity (CDS8659) with $A$. fumigatus has no iron-related genes in its vicinity; however, this ORF is clustered with genes involved in melanin biosynthesis, which requires the action of multicopper oxidases belonging to the laccases subfamily. These enzymes catalyze the oxidation of phenolic compounds that simultaneously converts $\mathrm{Fe}(\mathrm{III})$ to $\mathrm{Fe}(\mathrm{II})$, suggesting rather a role for CDS8659 in melanin production through a laccase (Fe(III)-reducing) activity. The two others $\mathrm{fetC} / \mathrm{ftrA}$ putative orthologs (CDS0314 and CDS0321, respectively) are separated by about $28 \mathrm{~kb}$. However, in fungi, these proteins are classically encoded by paired consecutive genes oriented on the opposite strand one from another (Kensche et al., 2008). The genes neighboring CDS0314 and CDS0321 therefore were analyzed through a BLASTp analysis against fungal genomic resources (taxid:4751). This allowed the identification of CDS0322 as a fetC ortholog, paired with the ftrA ortholog CDS0321, and of CDS0315 as an ftrA ortholog paired with the $f e t C$ ortholog CDS0314, thus revealing the 


\section{A}

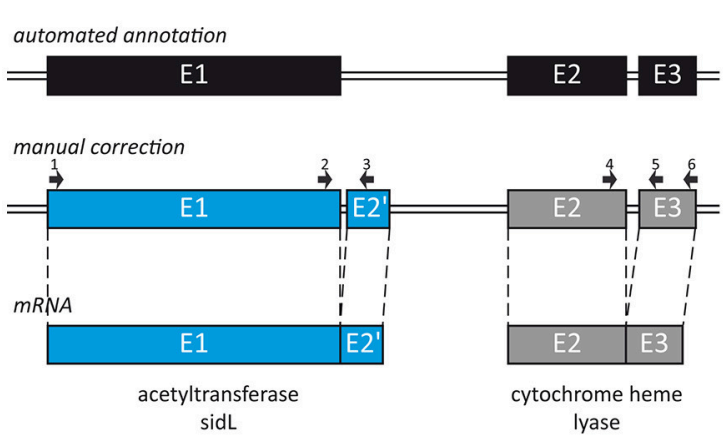

B

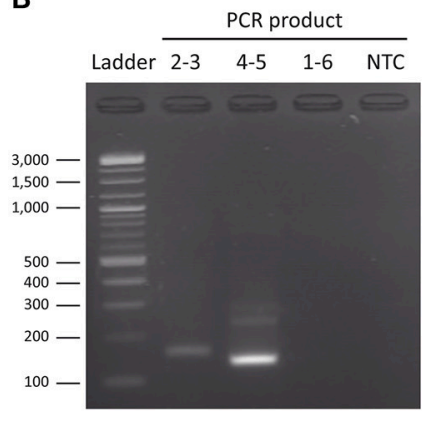

FIGURE 2 | Manual correction of ORF SAPIO_CDS2796 annotation. (A) To further refine the automated annotation performed on the genome of S. apiospermum IHEM 14462 (top), the size of the mRNA transcribed from CDS2796 was assessed by RT-PCR using 3 pairs of primers (middle): one pair spanning the newly discovered intron (2-3), another covering the last intron (4-5), and a third pair covering CDS2796 from start to stop codons as automatically annotated (1-6). (B) Gel electrophoresis analysis of the PCR products. The first primer pair (2-3) amplified an expected 123 bp-fragment, which confirms the existence of an intron at the end of E1. The second couple (4-5) also amplified a fragment of the expected size. On the opposite, the last pair of primers (1-6) gave no amplification, further confirming that the transcription of CDS2796 produces two mRNAs, the upstream one corresponding to a sidL ortholog (A, bottom). NTC, no-template control.

same tandem organization in $S$. apiospermum, None of the nine putative ferric reductases identified in the $S$. apiospermum genome was located in the vicinity of these two Ftr/Fet couples.

\section{Transcriptional Response According to Iron Availability}

To assess whether the genes predicted in silico were actually involved in iron homeostasis, we studied their expression in S. apiospermum cells grown for $48 \mathrm{~h}$ in iron starvation or iron excess conditions by qPCR (Figure 3). Globally, the variations of expression level observed in iron excess conditions were not influenced significantly by the source of iron $\left(\mathrm{FeCl}_{3}, \mathrm{FeSO}_{4}\right.$ or holotransferrine).

(i) Siderophore biosynthesis. The expression of siderophore biosynthesis-encoding genes, especially those involved in extracellular siderophore production (i.e., sidA, sidD, sidF, $s i d H$, and sidI orthologs), was highly induced under iron deprivation. By contrast, the genes involved in intracellular siderophore synthesis, i.e., sidC and sidL orthologs, as well as the putative $p p t A$ gene, which encodes a protein required for NRPSs activation (Allen et al., 2011), remained isoexpressed in our conditions. Besides, all of the siderophore genes that were overexpressed in iron-starved mycelia were downregulated during iron excess, except for the sidH ortholog, which remained up-regulated.

(ii) Ornithine metabolism. In $S$. apiospermum, the gene encoding the putative mitochondrial ornithine transporter $a m c A$ was up-regulated during iron starvation; by contrast, the arginase-encoding gene was barely expressed in all culture conditions tested. These results suggest that the cytosolic pool of ornithine is mainly fueled by mitochondria in S. apiospermum. (iii) Ferrisiderophores transport. As aforementioned, the S. apiospermum genome encodes a single sit $T$ and $8 \mathrm{mir}$ orthologs. In our conditions, the sit $T$ ortholog and 3 out of 8 mir orthologs (CDS2478, 4564, and 4736) were upregulated during iron starvation. Moreover, the expression of these four loci was downregulated in iron excess conditions, strongly suggesting their involvement in siderophoremediated iron uptake. Two other mir orthologs, CDS6391 and CDS9285, were strongly downregulated in ironoverloaded conditions. However, the increase in their expression level was not statistically significant during iron starvation (log2 fold-change \pm standard deviation: $0.33 \pm 0.50$ and $1.06 \pm 0.56$ for CDS6391 and CDS9285, respectively).

(iv) Reductive iron assimilation. Among the 9 putative ferric reductases found in the $S$. apiospermum genome, only the ortholog with the highest similarity to the A. fumigatus freB gene (CDS2383) was significantly down-regulated in iron-overloaded culture conditions. Four genes (CDS1476, 9014,10508 , and 10726) were not significantly expressed in any of the conditions tested, while another (CDS6952) was overexpressed under all assayed conditions. Moreover, only the RIA gene couple that displays the highest homology with the A. fumigatus fetC/ftrA gene cluster (CDS03140315) was induced under iron starvation. Besides, the ORF hypothesized to encode a laccase-type multicopper oxidase (CDS8659) was, as expected, unresponsive to all tested conditions.

(v) Vacuolar iron storage. Vacuolar sequestration probably occurs in S. apiospermum since the $c c c A$ homolog, which encodes a vacuolar iron importer in A. fumigatus (Gsaller et al., 2012), was significantly overexpressed during iron excess, while its expression remained unchanged under iron deprivation. 


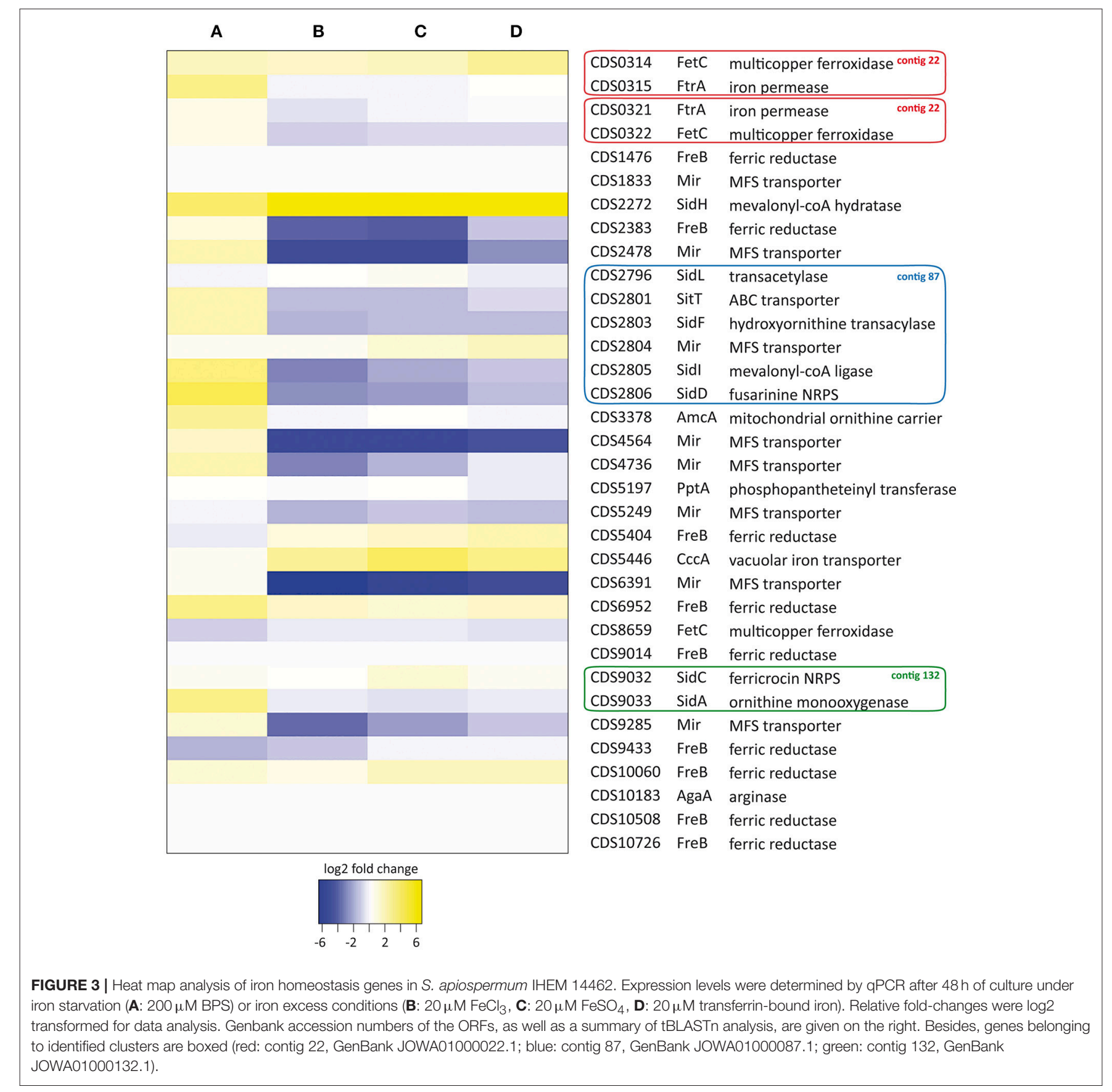

\section{DISCUSSION}

Iron is known to be metabolically essential for virtually all living organisms. Therefore, the "battle for iron" between a given pathogen and the host, but also between several pathogens coexisting within the same host (e.g., A. fumigatus and Pseudomonas aeruginosa in the CF lung), is a key determinant for a successful infection. To circumvent host-iron withholding, a number of bacterial and fungal pathogens have developed highaffinity iron uptake systems, some of which being mandatory for full virulence. Here, we investigated the presence of iron-related genes in S. apiospermum genome in order to find molecular mechanisms potentially underpinning pathogenicity.

The automated in silico analysis of S. apiospermum genome mis-annotated intronless genes as pseudogenes. Therefore the identification of genes putatively involved in iron metabolism was performed through a tBLASTn rather than a BLASTp analysis, using A. fumigatus Af293 iron-related proteins as query (Haas, 2012). This approach allowed to find orthologs for almost all genes involved in iron homeostasis in A. fumigatus. Furthermore, data mining revealed that most of the genes required for hydroxamate siderophore biosynthesis and 
transport were clustered in S. apiospermum. Such genomic organization has already been described in various filamentous fungi (Haas et al., 2008; Franken et al., 2014), but differs from one species to another (Figure 1). For instance, three distinct clusters have been identified in A. fumigatus and A. nidulans (Haas, 2012), whereas only two clusters are described in A. niger (Franken et al., 2014). One of these clusters contains sidC and sidI and seems to be preserved among the aforementioned three Aspergillus species. Interestingly, in S. apiospermum, the sidC-related cluster contains an ortholog of sidA instead of sidI (a feature also found in $T$. reesei and C. higginsianum), while sidA gene is not clustered in Aspergillus genomes. As for S. apiospermum sidI ortholog, it belongs to another cluster containing a series of genes putatively involved in siderophore biosynthesis (i.e., sidD, sidF, and sidL orthologs) and uptake (i.e., one sitT and one mir orthologs). In Aspergillus species, the sidD-related cluster also includes sidH, while in S. apiospermum the sidH ortholog is located in a different region of the genome together with a putative MFS transporter gene, and is separated from the sidD cluster by $110 \mathrm{~kb}$. Moreover, like sidA, sidL genes are not clustered with other siderophore-biosynthetic genes in Aspergillus genomes. Overall, comparative genomics showed that the $S$. apiospermum siderophore genes were organized as described in most of the siderophore-producing fungi including the aspergilli. Nevertheless, gene clustering in S. apiospermum was more similar to that observed in phylogenetically close phytopathogenic or mycoparasitic molds such as C. higginsianum and T. reesei.

Further analysis of the two $S$. apiospermum siderophoreassociated NRPS genes showed that the sidD ortholog encodes a protein with $44-45 \%$ sequence similarity with those produced by the three above-mentioned Aspergillus species. However, the precise structure of the synthesized metabolite could not be predicted on the single basis of the NRPS sequence. Indeed, although A. fumigatus and A. niger SidD are closely related (66\% identity), A. fumigatus produces the extracellular siderophore fusarinine $\mathrm{C}$, while $A$. niger synthesizes coprogen B (Franken et al., 2014). Besides, one of the closest ortholog of $A$. fumigatus SidD in non-Aspergillus species is found in Metarhizium robertsii. It displays $58 \%$ identity with S. apiospermum SidD ortholog and is involved in the biosynthesis of another coprogentype siderophore termed $N^{\alpha}$-dimethyl coprogen (Giuliano Garisto Donzelli et al., 2015). We previously demonstrated that $S$. apiospermum is able to synthesize and secrete the coprogen-type siderophore $N^{\alpha}$-methyl coprogen B (Bertrand et al., 2009). HPLC-MS also evidenced that S. apiospermum produces the dihydroxamate dimerumic acid, but its involvement in iron metabolism is controversial since it is both described as a breakdown product of coprogen and as a natural product of several molds like Verticillium dahliae or Penicillium chrysogenum (Donzelli and Krasnoff, 2016). Together, these data suggest that sidD is responsible for the biosynthesis of $N^{\alpha}$-methyl coprogen B in S. apiospermum.

Likewise, the prediction of the final non-ribosomal peptide synthesized by sidC orthologs is hazardous, if not impossible. For instance, experimental studies showed that despite the high degree of similarity existing among sidC within the Aspergillus genus, the most likely intracellular siderophore produced by A. niger is ferrichrome (Franken et al., 2014), while A. fumigatus and $A$. nidulans both synthesize ferricrocin (Haas et al., 2008). Until now, only extracellular siderophores have been identified in S. apiospermum. Nevertheless, ferricrocin has been detected in the closely related species $S$. boydii (Vladimír Havlíček, personal communication), in which the putative sidC gene encodes a protein that shares 93\% identity with XP_016639834.1 encoded by S. apiospermum sidC ortholog (CDS9032). Thus, even if massspectrometry analyses are needed, it is highly probable that $S$. apiospermum also produces ferricrocin.

Expression data showed that the $S$. apiospermum genes involved in extracellular siderophores biosynthesis (i.e., sidA, sidD, sidF, sidH, and sidI orthologs) were significantly induced during iron starvation. Conversely, expression of the genes specifically implicated in intracellular siderophores production, i.e., sidC and sidL orthologs, as well as the NRPS activator gene $p p t A$, remained stable in this condition. The last 2 genes are known to be constitutively expressed in A. fumigatus (Oberegger et al., 2003; Blatzer et al., 2011c). More surprising is the unchanged expression level of the sidC ortholog, since previous studies based on Northern-blot analyses in Aspergillus showed that transcription of this gene was detectable only during iron starvation (Oberegger et al., 2002; Eisendle et al., 2003; Schrettl et al., 2007). However, more recent studies showed that sidC expression is only weakly affected by low iron concentrations (i.e., $\leq 20 \mu \mathrm{M}$ ) in both Aspergillus and non-Aspergillus species (Reiber et al., 2005; López-Berges et al., 2012; Franken et al., 2014). The iron concentration in our experimental conditions $(20 \mu \mathrm{M})$ could be insufficient to induce significant overexpression of sidC. Likewise, compared to Aspergillus species, S. apiospermum is a slow-growing fungus and one can hypothesize that sidC overexpression is time-delayed in this species. On the other hand, all siderophore genes that were overexpressed in iron starved mycelia were down-regulated during iron excess, except for the sidH ortholog, which remained up-regulated. Given that the sidH-encoded enoyl-CoA hydratase catalyzes a reversible reaction (Abdel-Mawgoud et al., 2013), one may speculate that its overexpression in iron excess may help to drop off the peroxisomal pathway and to diminish the production of extracellular siderophores in iron-rich environments.

Among the 9 putative SIT (1 sitT and 8 mir) orthologs found in S. apiospermum genome, one mir ortholog (CDS2804) and the sit $T$ ortholog (CDS2801) are part of the sidD-related cluster, a feature also observed in Aspergillus spp. and T. reesei. The expression of the $S$. apiospermum putative sitT and of 3 out of the 8 mir orthologs (CDS2478, CDS4564, and CDS4736) varies with iron availability, indicating that they probably participate in ferrisiderophore uptake. Strikingly, the mir ortholog belonging to the sidD cluster, as well as the four remaining genes, was not statistically overexpressed under iron depletion. In A. fumigatus, iron starvation induces iron-related genes transcription by the recruitment of the HapX transcription factor at CCAAT sequences present in their promoter, through the interaction of HapX with the CCAAT-binding complex (CBC) (Hortschansky et al., 2007). Promoter analysis of four SIT encoding genes responsive to iron starvation (CDS2801, 2478, 4564, 4736) 
revealed the presence of 3 to 5 CCAAT motifs while only one CCAAT motif was found in the promoter of the unresponsive transporter gene CDS2804 (Supplementary Table S2). Thus, we first hypothesized that the level of expression of SIT genes could be related to the number of CCAAT motifs present in their promoter. Nevertheless, we also found 2 to 5 CCAAT motifs in the promoter of mir orthologs unresponsive to iron starvation (CDS1833, 5249, 6391, and 9285). Of note, 3 out of these 4 Mir-encoding genes (CDS1833, 6391, and 9285) belong to gene clusters also organized around some NRPS encoding genes (CDS1828, 6390 and 9291, respectively), but totally unrelated to iron metabolism (unpublished results). The absence of induction of the mir orthologs CDS2804 and 5249 remains to be explained.

Despite an apparent expansion of the gene set putatively involved in RIA $(n=14)$, only three (CDS2383, 0314, and 0315) showed adequate response to the tested conditions, i.e., up-regulation during iron starvation and/or down-regulation during iron excess. RIA is a tripartite system made of one metalloreductase associated with a ferroxidase/ferripermease tandem. Aspergillus fumigatus genome harbors 15 putative metalloreductase genes, but only one, namely freB, is involved in iron metabolism (Blatzer et al., 2011b). Transcriptional analysis showed that the expression of freB was repressed by the GATA transcription factor SreA during iron sufficiency. Interestingly, the only putative $S$. apiospermum ferric-reductase gene that was down-regulated by iron corresponded to the best hit with the A. fumigatus ortholog (CDS2383). Likewise, only the RIA gene cluster showing the highest degree of homology with the A. fumigatus fetC/ftrA gene pair was significantly overexpressed during iron starvation (CDS0314/CDS0315). Of note, two FetC/FtrA homologs with distinct functions are described in the yeast Saccharomyces cerevisiae; indeed the Fet $3 \mathrm{p} / \mathrm{Ftr} 1 \mathrm{p}$ complex mediates $\mathrm{Fe}(\mathrm{III})$ channeling in the yeast plasma membrane, while the paralog Fet5p/Fth1p complex mediates iron moves from the yeast vacuole (Urbanowski and Piper, 1999). Consequently,

\section{REFERENCES}

Abdel-Mawgoud, A. M., Lépine, F., and Déziel, E. (2013). A chiral highperformance liquid chromatography-tandem mass spectrometry method for the stereospecific analysis of enoyl-coenzyme A hydratases/isomerases. J. Chromatogr. A 1306, 37-43. doi: 10.1016/j.chroma.2013.07.049

Allen, G., Bromley, M., Kaye, S. J., Keszenman-Pereyra, D., Zucchi, T. D., Price, J., et al. (2011). Functional analysis of a mitochondrial phosphopantetheinyl transferase (PPTase) gene pptB in Aspergillus fumigatus. Fungal Genet. Biol. 48, 456-464. doi: 10.1016/j.fgb.2010.12.006

Bertrand, S., Larcher, G., Landreau, A., Richomme, P., Duval, O., and Bouchara, J. P. (2009). Hydroxamate siderophores of Scedosporium apiospermum. Biometals 22, 1019-1029. doi: 10.1007/s10534-009-9253-0

Blatzer, M., Barker, B. M., Willger, S. D., Beckmann, N., Blosser, S. J., Cornish, E. J., et al. (2011a). SREBP coordinates iron and ergosterol homeostasis to mediate triazole drug and hypoxia responses in the human fungal pathogen Aspergillus fumigatus. PLoS Genet. 7:e1002374. doi: 10.1371/journal.pgen.1002374

Blatzer, M., Binder, U., and Haas, H. (2011b). The metalloreductase FreB is involved in adaptation of Aspergillus fumigatus to iron starvation. Fungal Genet. Biol. 48, 1027-1033. doi: 10.1016/j.fgb.2011.07.009

Blatzer, M., Schrettl, M., Sarg, B., Lindner, H. H., Pfaller, K., and Haas, H. (2011c). SidL, an Aspergillus fumigatus transacetylase involved in biosynthesis of the one may hypothesize that the non-responsive S. apiospermum fetC/ftrA gene cluster is involved in vacuolar trafficking of iron rather than in RIA. Moreover, we could identify an iron transporter $c c c A$ ortholog in the genome, the expression of which was significantly induced in iron-overloaded cells, suggesting that a vacuolar iron homeostasis system exists in S. apiospermum.

Altogether, these findings indicate that $S$. apiospermum possesses genetic information needed for iron uptake and regulation. Expression data suggest that, in mycelia, iron acquisition is mediated by both RIA and the siderophore system. Our research group already evidenced the production of extracellular siderophore in S. apiospermum, and our genomic analysis found putative orthologous genes for both extra- and intracellular siderophore biosynthesis. Works are in progress to identify all the hydroxamate-type siderophores produced by S. apiospermum, with a particular emphasis on intracellular siderophore biosynthesis. Moreover, the role of siderophores during Scedosporium infections has not been studied so far, and experiments in a rodent model of scedosporiosis are planned to evaluate their implication in Scedosporium virulence.

\section{AUTHOR CONTRIBUTIONS}

YL, J-PB, PV: Conceived and designed the experiments; YL, PV: Performed the experiments; YL, NP, SL, BL, PV: Analyzed the data; YL, PV: Wrote the paper. All authors read and approved the manuscript.

\section{SUPPLEMENTARY MATERIAL}

The Supplementary Material for this article can be found online at: https://www.frontiersin.org/articles/10.3389/fmicb. 2018.00827/full\#supplementary-material siderophores ferricrocin and hydroxyferricrocin. Appl. Environ. Microbiol. 77, 4959-4966. doi: 10.1128/AEM.00182-11

Chen, M., Kondori, N., Deng, S., Gerrits van den Ende, A. H. G., Lackner, M., Liao, W., et al. (2017). Direct detection of Exophiala and Scedosporium species in sputa of patients with cystic fibrosis. Med. Mycol. doi: 10.1093/mmy/myx108. [Epub ahead of print].

Cortez, K. J., Roilides, E., Quiroz-Telles, F., Meletiadis, J., Antachopoulos, C., Knudsen, T., et al. (2008). Infections caused by Scedosporium spp. Clin. Microbiol. Rev. 21, 157-197. doi: 10.1128/CMR. 00039-07

Donzelli, B. G. G., and Krasnoff, S. B. (2016). "Chapter ten - Molecular genetics of secondary chemistry in metarhizium fungi," in Advances in Genetics Genetics and Molecular Biology of Entomopathogenic Fungi, eds B. Lovett and R. J. St. Leger (Academic Press), 365-436.

Douglas, A. P., Chen, S. C., and Slavin, M. A. (2016). Emerging infections caused by non-Aspergillus filamentous fungi. Clin. Microbiol. Infect. 22, 670-680. doi: 10.1016/j.cmi.2016.01.011

Eisendle, M., Oberegger, H., Zadra, I., and Haas, H. (2003). The siderophore system is essential for viability of Aspergillus nidulans: functional analysis of two genes encoding l-ornithine N 5-monooxygenase (sidA) and a non-ribosomal peptide synthetase (sidC). Mol. Microbiol. 49, 359-375. doi: 10.1046/j.1365-2958.2003.03586.x 
Franken, A. C., Lechner, B. E., Werner, E. R., Haas, H., Lokman, B. C., Ram, A. F., et al. (2014). Genome mining and functional genomics for siderophore production in Aspergillus niger. Brief. Funct. Genomics 13, 482-492. doi: 10.1093/bfgp/elu026

Frey, P. A., and Reed, G. H. (2012). The ubiquity of iron. ACS Chem. Biol. 7, 1477-1481. doi: 10.1021/cb300323q

Galagan, J. E., Calvo, S. E., Cuomo, C., Ma, L.-J., Wortman, J. R., Batzoglou, S., et al. (2005). Sequencing of Aspergillus nidulans and comparative analysis with A. fumigatus and A. oryzae. Nature 438, 1105-1115. doi: 10.1038/nature04341

Giuliano Garisto Donzelli, B., Gibson, D. M., and Krasnoff, S. B. (2015). Intracellular siderophore but not extracellular siderophore is required for full virulence in Metarhizium robertsii. Fungal Genet. Biol. 82, 56-68. doi: 10.1016/j.fgb.2015.06.008

Grant, C. E., Bailey, T. L., and Noble, W. S. (2011). FIMO: scanning for occurrences of a given motif. Bioinformatics 27, 1017-1018. doi: 10.1093/bioinformatics/btr064

Gründlinger, M., Yasmin, S., Lechner, B. E., Geley, S., Schrettl, M., Hynes, M., et al. (2013). Fungal siderophore biosynthesis is partially localized in peroxisomes. Mol. Microbiol. 88, 862-875. doi: 10.1111/mmi.12225

Gsaller, F., Eisendle, M., Lechner, B. E., Schrettl, M., Lindner, H., Müller, D., et al. (2012). The interplay between vacuolar and siderophore-mediated iron storage in Aspergillus fumigatus. Metallomics 4, 1262-1270. doi: 10.1039/c2mt20179h

Guarro, J., Kantarcioglu, A. S., Horr,é, R., Rodriguez-Tudela, J. L., Cuenca Estrella, M., Berenguer, J., et al. (2006). Scedosporium apiospermum: changing clinical spectrum of a therapy-refractory opportunist. Med. Mycol. 44, 295-327. doi: 10.1080/13693780600752507

Haas, H. (2012). Iron - a key nexus in the virulence of Aspergillus fumigatus. Front. Microbiol. 3:28. doi: 10.3389/fmicb.2012.00028

Haas, H. (2014). Fungal siderophore metabolism with a focus on Aspergillus fumigatus. Nat. Prod. Rep. 31, 1266-1276. doi: 10.1039/C4NP00071D

Haas, H., Eisendle, M., and Turgeon, B. G. (2008). Siderophores in fungal physiology and virulence. Annu. Rev. Phytopathol. 46, 149-187. doi: 10.1146/annurev.phyto.45.062806.094338

Haas, H., Schoeser, M., Lesuisse, E., Ernst, J. F., Parson, W., Abt, B., et al. (2003). Characterization of the Aspergillus nidulans transporters for the siderophores enterobactin and triacetylfusarinine C. Biochem. J. 371, 505-513. doi: $10.1042 /$ bj20021685

Halliwell, B., and Gutteridge, J. M. (1984). Oxygen toxicity, oxygen radicals, transition metals and disease. Biochem. J. 219, 1-14. doi: 10.1042/bj2190001

Hortschansky, P., Eisendle, M., Al-Abdallah, Q., Schmidt, A. D., Bergmann, S., Thön, M., et al. (2007). Interaction of HapX with the CCAAT-binding complex-a novel mechanism of gene regulation by iron. EMBO J. 26, 3157-3168. doi: 10.1038/sj.emboj.7601752

Jung, W. H., Sham, A., Lian, T., Singh, A., Kosman, D. J., and Kronstad, J. W. (2008). Iron source preference and regulation of iron uptake in Cryptococcus neoformans. PLoS Pathog. 4:e45. doi: 10.1371/journal.ppat.0040045

Kensche, P. R., Oti, M., Dutilh, B. E., and Huynen, M. A. (2008). Conservation of divergent transcription in fungi. Trends Genet. TIG 24, 207-211. doi: 10.1016/j.tig.2008.02.003

Knight, S. A., Vilaire, G., Lesuisse, E., and Dancis, A. (2005). Iron acquisition from transferrin by Candida albicans depends on the reductive pathway. Infect. Immun. 73, 5482-5492. doi: 10.1128/IAI.73.9.5482-5492.2005

Koehler, P., Tacke, D., and Cornely, O. A. (2016). Bone and joint infections by Mucorales, Scedosporium, Fusarium and even rarer fungi. Crit. Rev. Microbiol. 42, 158-171. doi: 10.3109/1040841X.2014.910749

Kosman, D. J. (2013). Iron metabolism in aerobes: managing ferric iron hydrolysis and ferrous iron autoxidation. Coord. Chem. Rev. 257, 210-217. doi: 10.1016/j.ccr.2012.06.030

Kragl, C., Schrettl, M., Abt, B., Sarg, B., Lindner, H. H., and Haas, H. (2007). EstB-mediated hydrolysis of the siderophore triacetylfusarinine C optimizes iron uptake of Aspergillus fumigatus. Eukaryotic Cell 6, 1278-1285. doi: 10.1128/EC.00066-07

Lamaris, G. A., Chamilos, G., Lewis, R. E., Safdar, A., Raad, I. I., and Kontoyiannis, D. P. (2006). Scedosporium infection in a tertiary care cancer center: a review of 25 cases from 1989-2006. Clin. Infect. Dis. 43, 1580-1584. doi: 10.1086/509579

Llanos, A., François, J. M., and Parrou, J. L. (2015). Tracking the best reference genes for RT-qPCR data normalization in filamentous fungi. BMC Genomics 16:71. doi: 10.1186/s12864-015-1224-y
López-Berges, M. S., Capilla, J., Turrà, D., Schafferer, L., Matthijs, S., Jöchl, C., et al. (2012). HapX-mediated iron homeostasis is essential for rhizosphere competence and virulence of the soilborne pathogen Fusarium oxysporum. Plant Cell 24, 3805-3822. doi: 10.1105/tpc.112.098624

Martinez, D., Berka, R. M., Henrissat, B., Saloheimo, M., Arvas, M., Baker, S. E., et al. (2008). Genome sequencing and analysis of the biomass-degrading fungus Trichoderma reesei (syn. Hypocrea jecorina). Nat. Biotechnol. 26, 553-560. doi: 10.1038/nbt1403

Nierman, W. C., Pain, A., Anderson, M. J., Wortman, J. R., Kim, H. S., Arroyo, J., et al. (2005). Genomic sequence of the pathogenic and allergenic filamentous fungus Aspergillus fumigatus. Nature 438, 1151-1156. doi: 10.1038/nature04332

Oberegger, H., Eisendle, M., Schrettl, M., Graessle, S., and Haas, H. (2003). $4^{\prime}$-phosphopantetheinyl transferase-encoding npgA is essential for siderophore biosynthesis in Aspergillus nidulans. Curr. Genet. 44, 211-215. doi: 10.1007/s00294-003-0434-z

Oberegger, H., Zadra, I., Schoeser, M., Abt, B., Parson, W., and Haas, H. (2002). Identification of members of the Aspergillus nidulans SREA regulon: genes involved in siderophore biosynthesis and utilization. Biochem. Soc. Trans. 30, 781-783. doi: 10.1042/bst0300781

O’Connell, R. J., Thon, M. R., Hacquard, S., Amyotte, S. G., Kleemann, J., Torres, M. F., et al. (2012). Lifestyle transitions in plant pathogenic Colletotrichum fungi deciphered by genome and transcriptome analyses. Nat. Genet. 44, 1060-1065. doi: 10.1038/ng.2372

Pel, H. J., de Winde, J. H., Archer, D. B., Dyer, P. S., Hofmann, G., Schaap, P. J., et al. (2007). Genome sequencing and analysis of the versatile cell factory Aspergillus niger CBS 513.88. Nat. Biotechnol. 25, 221-231. doi: 10.1038/nbt1282

Philpott, C. C. (2006). Iron uptake in fungi: a system for every source. Biochim. Biophys. Acta 1763, 636-645. doi: 10.1016/j.bbamcr.2006.05.008

Pihet, M., Carrere, J., Cimon, B., Chabasse, D., Delhaes, L., Symoens, F., et al. (2009). Occurrence and relevance of filamentous fungi in respiratory secretions of patients with cystic fibrosis-a review. Med. Mycol. 47, 387-397. doi: 10.1080/13693780802609604

Ramanan, N., and Wang, Y. (2000). A high-affinity iron permease essential for Candida albicans virulence. Science 288, 1062-1064. doi: $10.1126 /$ science.288.5468.1062

Reiber, K., Reeves, E. P., Neville, C. M., Winkler, R., Gebhardt, P., Kavanagh, K., et al. (2005). The expression of selected non-ribosomal peptide synthetases in Aspergillus fumigatus is controlled by the availability of free iron. FEMS Microbiol. Lett. 248, 83-91. doi: 10.1016/j.femsle.2005.05.028

Saikia, S., Oliveira, D., Hu, G., and Kronstad, J. (2014). Role of ferric reductases in iron acquisition and virulence in the fungal pathogen Cryptococcus neoformans. Infect. Immun. 82, 839-850. doi: 10.1128/IAI.01357-13

Schafferer, L., Beckmann, N., Binder, U., Brosch, G., and Haas, H. (2015). AmcAa putative mitochondrial ornithine transporter supporting fungal siderophore biosynthesis. Front. Microbiol. 6:252. doi: 10.3389/fmicb.2015.00252

Schrettl, M., Beckmann, N., Varga, J., Heinekamp, T., Jacobsen, I. D., Jöchl, C., et al. (2010). HapX-mediated adaption to iron starvation is crucial for virulence of Aspergillus fumigatus. PLoS Pathog. 6:e1001124. doi: 10.1371/journal.ppat.1001124

Schrettl, M., Bignell, E., Kragl, C., Joechl, C., Rogers, T., Arst, H. N., et al. (2004). Siderophore biosynthesis but not reductive iron assimilation is essential for Aspergillus fumigatus virulence. J. Exp. Med. 200, 1213-1219. doi: $10.1084 /$ jem.20041242

Schrettl, M., Bignell, E., Kragl, C., Sabiha, Y., Loss, O., Eisendle, M., et al. (2007). Distinct roles for intra- and extracellular siderophores during Aspergillus fumigatus infection. PLoS Pathog. 3, 1195-1207. doi: 10.1371/journal.ppat.0030128

Schrettl, M., Kim, H. S., Eisendle, M., Kragl, C., Nierman, W. C., Heinekamp, T., et al. (2008). SreA-mediated iron regulation in Aspergillus fumigatus. Mol. Microbiol. 70, 27-43. doi: 10.1111/j.1365-2958.2008.06376.x

Stearman, R., Yuan, D. S., Yamaguchi-Iwai, Y., Klausner, R. D., and Dancis, A. (1996). A permease-oxidase complex involved in high-affinity iron uptake in yeast. Science 271, 1552-1557. doi: 10.1126/science.271.5255.1552

Urbanowski, J. L., and Piper, R. C. (1999). The iron transporter Fth1p forms a complex with the Fet5 iron oxidase and resides on the vacuolar membrane. J. Biol. Chem. 274, 38061-38070. doi: 10.1074/jbc.274.53.38061

Vandeputte, P., Ghamrawi, S., Rechenmann, M., Iltis, A., Giraud, S., Fleury, M., et al. (2014). Draft genome sequence of the pathogenic 
fungus Scedosporium apiospermum. Genome Announc. 2:e00988-14. doi: 10.1128/genomeA.00988-14

von Döhren, H. (2009). A survey of nonribosomal peptide synthetase (NRPS) genes in Aspergillus nidulans. Fungal Genet. Biol. 46(Suppl. 1), S45-S52. doi: 10.1016/j.fgb.2008.08.008

Walsh, T. J., and Groll, A. H. (1999). Emerging fungal pathogens: evolving challenges to immunocompromised patients for the twenty-first century. Transpl. Infect. Dis. 1, 247-261. doi: 10.1034/j.1399-3062.1999.010404.x

Wang, T. P., Quintanar, L., Severance, S., Solomon, E. I., and Kosman, D. J. (2003). Targeted suppression of the ferroxidase and iron trafficking activities of the multicopper oxidase Fet3p from Saccharomyces cerevisiae. J. Biol. Inorg. Chem. 8, 611-620. doi: 10.1007/s00775-003-0456-5

Yasmin, S., Alcazar-Fuoli, L., Gründlinger, M., Puempel, T., Cairns, T., Blatzer, M., et al. (2012). Mevalonate governs interdependency of ergosterol and siderophore biosyntheses in the fungal pathogen Aspergillus fumigatus. Proc. Natl. Acad. Sci. U.S.A. 109, E497-E504. doi: 10.1073/pnas.1106399108
Yun, C. W., Bauler, M., Moore, R. E., Klebba, P. E., and Philpott, C. C. (2001). The role of the FRE family of plasma membrane reductases in the uptake of siderophore-iron in Saccharomyces cerevisiae. J. Biol. Chem. 276, 10218-10223. doi: 10.1074/jbc.M010065200

Conflict of Interest Statement: The authors declare that the research was conducted in the absence of any commercial or financial relationships that could be construed as a potential conflict of interest.

Copyright (C) 2018 Le Govic, Papon, Le Gal, Lelièvre, Bouchara and Vandeputte. This is an open-access article distributed under the terms of the Creative Commons Attribution License (CC BY). The use, distribution or reproduction in other forums is permitted, provided the original author(s) and the copyright owner are credited and that the original publication in this journal is cited, in accordance with accepted academic practice. No use, distribution or reproduction is permitted which does not comply with these terms. 